\title{
Dysphagia and Eosinophilic Infiltration of the Esophageal Muscular Layer: A Case Report
}

\author{
Jose Francisco Plaz ${ }^{\mathrm{a}}$, Renzo Giuseppe Di Natale ${ }^{\mathrm{b}, \mathrm{c}}$, Marisa Di Natale ${ }^{\mathrm{b}}$, Andres Javier Gonzalez-Salazar ${ }^{\mathrm{b}}$, \\ Raquel Alexandra Lander ${ }^{\mathrm{b}}$
}

\begin{abstract}
Eosinophilic gastrointestinal diseases are a group of pathological entities characterized by an eosinophilic infiltration of the gastrointestinal tract without known cause. Eosinophilic esophagitis (EE) is one of these conditions, which has three diagnostic criteria: symptoms of esophageal dysfunction, mucosal infiltration of 15 eosinophils per high-power field (HPF) microscope and no response to high-dose proton pump inhibitors (PPIs). A 51-year-old patient attended to the emergency department claiming to suffer from heartburn, dysphagia, regurgitation and chest pain. He was treated with high doses of PPIs without success and the condition progressed to food impaction. An esophagogram, which showed esophageal motor dysfunction in the middle and lower part of the esophagus, was performed, and pathologic examination of an esophageal biopsy taken by endoscopy revealed histopathological changes suggestive of gastroesophageal reflux disease. An esophageal manometry was carried out and it reported diffuse esophageal spasms and a hypertensive inferior esophageal sphincter with incomplete relaxation. A Heller cardiomyotomy was performed and the biopsy specimen revealed fibrosis and eosinophilic infiltration of the muscular layer. Symptoms ceased for 6 months after which symptoms reappeared. The patient was diagnosed with EE and treated with budesonide orally $12 \mathrm{mg} /$ day with complete remission of all symptoms. In this peculiar case, the eosinophilic infiltration was in the muscular layer of the esophagus, not in the mucosa; therefore, it did not meet the diagnostic criteria of EE nor its differential diagnoses. Nevertheless, treatment with oral steroids was in fact effective.
\end{abstract}

Keywords: Dysphagia; Esophagitis; Eosinophilia; Muscular; Infiltration

Manuscript accepted for publication September 08, 2014

${ }^{a}$ Centro Medico de Caracas, Caracas, Venezuela

b"Luis Razetti" School of Medicine, Universidad Central de Venezuela, Caracas, Venezuela

${ }^{\mathrm{c} C o r r e s p o n d i n g ~ A u t h o r: ~ R e n z o ~ G i u s e p p e ~ D i ~ N a t a l e, ~ I n s t i t u t o ~ d e ~ O r t o d o n c i a ~}$ Chuao, Calle Choroni, Quinta Ana Teresa, Chuao, PC: 1071, Caracas, Distrito Capital, Venezuela. Email: renzogdg@gmail.com

doi: http://dx.doi.org/10.14740/jmc1942w

\section{Introduction}

Eosinophilic esophagitis (EE) was a rarely identified disease until the last decade of the 20th century, when in 1993 it was first described as a clinical entity of its own. The case consisted of a patient with dysphagia and eosinophilic infiltration of the esophagus mucosa [1].

Current epidemiological studies suggest that this disease is more common than previously thought. Noel et al demonstrated that the incidence of the disease in Cincinnati, $\mathrm{OH}$, was 10 out of 100,000 children and the prevalence was 43 out of 100,000 [2]. In Australia, the incidence of primary EE increased from 0.05 to 0.89 out of 10,000 between 1995 and 2004 [3]. Nevertheless, it is unknown if this rise is due to a real incidence increase or just better diagnosis.

EE is part of a group of pathological entities that have eosinophilic infiltration; these are known as gastrointestinal eosinophilic diseases (GEDs). There are various theories that try to explain the physiopathological process of sensitization and activation of eosinophils in the esophagus. The first theory consists on an exposure of the bronchi to an aeroallergen, which consequently extends to the esophagus forming an aeroesophageal immune unit [4]. Other theories relate EE to allergen ingestion through food, producing organ sensitization. Blanchard et al demonstrated that patients with EE have an induction of the eotaxin-3 gene (CCL26) which has a chemoattractant effect on eosinophils. They determined that levels of this gene expression directly correlate to the severity of the disease taking into account the number of eosinophils and mast cells as well as their basal layer expansion [5].

EE affects predominantly men between the ages of 20 to 40 on a 3:1 male-to-female ratio. More than $90 \%$ of patients with EE present with intermittent dysphagia to solids which could lead to food impaction on the esophagus. They can also present heartburn, non-cardiac chest pain or abdominal pain and vomiting [6].

Eighty percent of patients with EE have a history of atopic disease. They can also present peripheral eosinophilia and increased serum immunoglobulin E levels [7].

Endoscopic alterations of EE consist on longitudinal or annular furrows on a fragile, corrugated esophageal mucosa. Other findings are narrowness, white papules and a diminished caliber on the middle and distal esophagus. Distal erosive modifications, typical of gastroesophageal reflux disease 


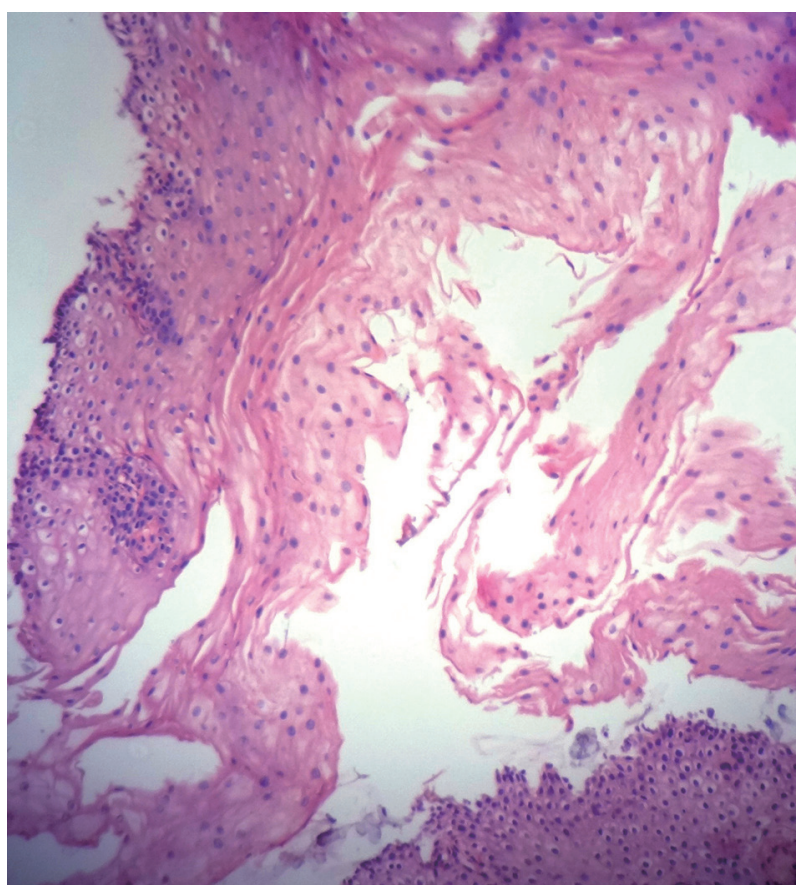

Figure 1. Biopsy of an endoscopically obtained sample of the esophagus $(\times 10)$. Note the absence of eosinophils infiltrating the mucosal epithelium.

(GERD), are not present [8].

EE diagnosis must meet the following criteria: symptoms of esophageal dysfunction, more than 15 eosinophils per highpower field (HPF) on the esophageal mucosa and no response to high doses of proton pump inhibitors (PPIs) or normal $\mathrm{pH}$ monitoring results on the distal esophagus [6].

\section{Case Report}

A 51-year-old male patient, who suffered from hypertension (treatment with amlodipine $5 \mathrm{mg}$ PO bid), presented with heartburn, dysphagia, regurgitation and retrosternal pain to the emergency department. An upper digestive endoscopy was performed, which reported hiatal hernia and characteristic changes of non-erosive GERD; the stomach and duodenum revealed no abnormalities. The mucosal biopsy of the esophagus showed histopathological changes suggestive of GERD (Fig. 1). Colonoscopy was also performed at this time, and it did not reveal any abnormalities. Biopsy samples were not taken from other organs (e.g. stomach, duodenum and colon), as no evidence of alterations was observed on endoscopic studies. The patient was therefore diagnosed with GERD and treatment with esomeprazole and ranitidine was started at high doses. A complete blood count performed at this time showed an eosinophil count of $4 \%$ (eosinophil absolute count (EAC): 580 eosinophils $/ \mu \mathrm{L}$ ).

During the following 3 months, symptoms worsened which resulted in a 30-kg weight loss. The patient was admitted to the emergency care unit. The eosinophil count at this moment was 1.0\% (EAC: 159 eosinophils $/ \mu \mathrm{L}$ ). An esophagogram showed

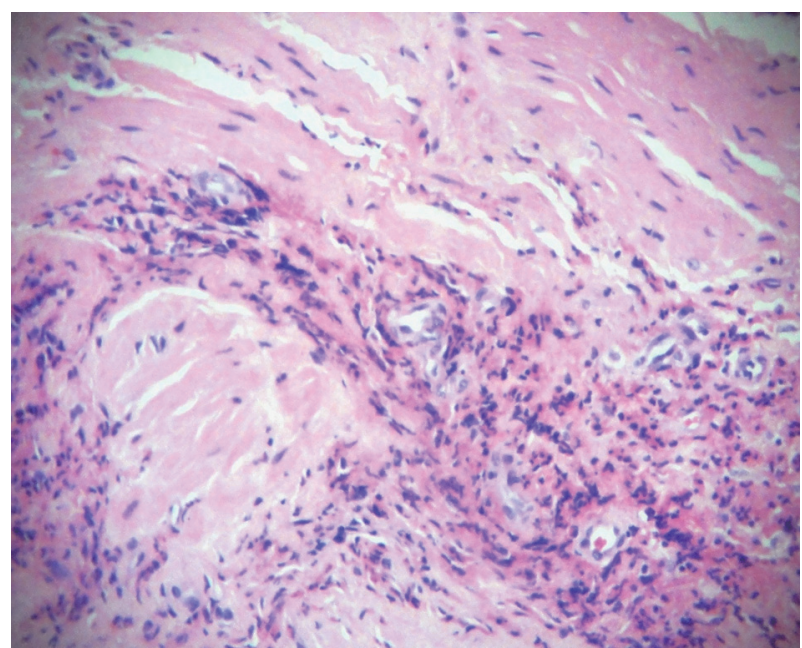

Figure 2. Biopsy of the muscular layer sample obtained during the Heller cardiomyotomy $(\times 30)$. Note the presence of a mixed-cell inflammatory infiltrate, composed primarily by eosinophils.

esophageal motor dysfunction and scarce aperture of the mid and distal esophagus. An esophageal manometry was also performed and reported diffuse esophageal spasm and lower esophageal sphincter (LES) hypertension with incomplete relaxation. Due to these results achalasia was diagnosed and a laparoscopic Heller cardiomyotomy was carried out. During the procedure, biopsy samples were taken from the esophageal mucosa, muscular layer and paraesophageal fat.

The biopsy demonstrated an extensive esophageal wall fibrosis with an inflammatory mixed-cell infiltrate composed primarily by eosinophils, which comprised the muscular layer (Fig. 2, 3). Treatment with esomeprazol $40 \mathrm{mg}$ bid and prednisone $50 \mathrm{mg}$ was indicated for 3 months with apparent reduction of symptoms.

During this time, different diagnostic procedures were carried out. A CBC after discharge revealed an eosinophil count of $12.2 \%$ (EAC: 1,172 eosinophils $/ \mu \mathrm{L}$ ). A skin-pick test (which showed no sensitization to any antigens) and determination of IgE levels were performed and showed no abnormalities (IgE: $87.3 \mathrm{IU} / \mathrm{mL}$, reference values: 20.00 - $100.00 \mathrm{IU} / \mathrm{mL}$ ). Celiac disease antibodies measured at this time were negative. Stool was examined in various opportunities but there was no evidence of bacterial or parasitic infection. A video capsule endoscopy was performed and it revealed no abnormalities in the gastrointestinal tract.

One month later, the patient presented to the emergency department because his previous symptoms had resurfaced. During the interrogation the patient referred having a papulous exanthema on his face and neck before the symptoms appeared which was treated with topical cetirizine. Macular erythematous lesions of the skin were seen on his face. He also referred moderate pain during epigastric palpation.

A superior digestive endoscopy was performed and reported a normal-aspect mucosa, a stomach with abundant food residues in the body and fundus, and an edematous pylorus and duodenum. Presence of fragments of esophageal squamous epithelium with discrete acanthosis and papillomatosis and 


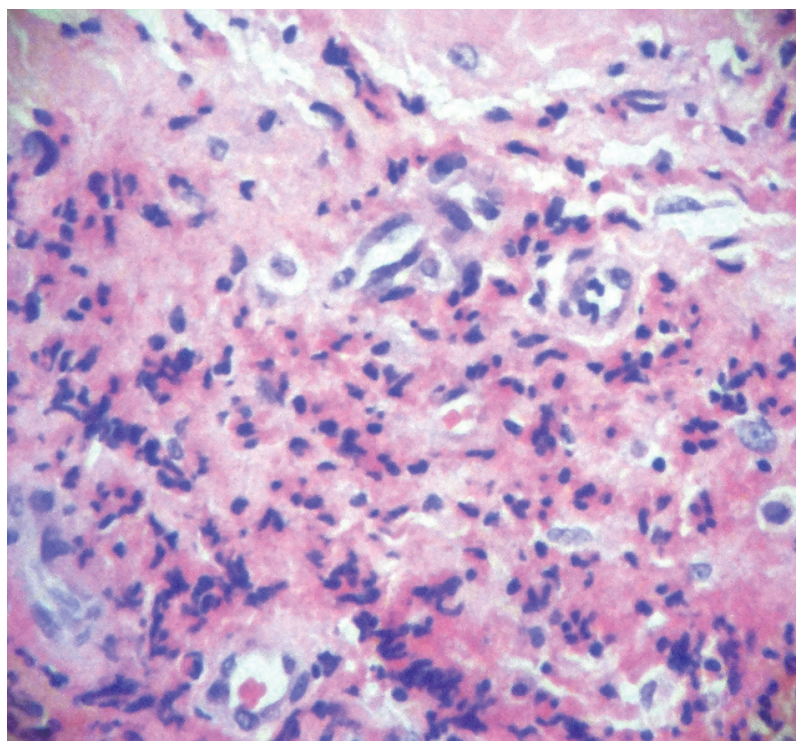

Figure 3. Biopsy of the muscular layer sample obtained during the Heller cardiomyotomy $(\times 100)$. Note the abundance of eosinophils infiltrating the muscular fibers.

chronic gastritis in the antrum (with focal epithelial loss and necrosis of the superior third of the mucosa) were also noticed on histopathological analysis.

Owing to the persistence of symptoms, a double-contrast computed tomography (CT) scan of the thorax, abdomen and pelvis was performed. A diffuse concentric thickening of the mid and lower esophagus conditioned a small hydro-aerial level. The esophageal alteration extended throughout the esophagogastric junction to the gastric walls, which had a $1.9 \mathrm{~cm}$ thickening in the gastric fundus (Fig. 4).

During his hospitalization, the patient received methylprednisolone $250 \mathrm{mg}$ IV bid with significant improvement 24 $\mathrm{h}$ after the initial dose. He was discharged 2 days later with oral methylprednisolone, indicated for 2 months. Eosinophil count at discharge was $4.6 \%$ (EAC: 515 eosinophils $/ \mu \mathrm{L}$ ) (Fig. $5)$. The methylprednisolone was later replaced with oral budesonide $12 \mathrm{mg}$ OD for 4 months and dose was later reduced to $9 \mathrm{mg}$ OD.

A control CT scan of the thorax (with oral contrast) was performed 4 months after discharge, and showed diffuse thickening of the esophagus walls from the level of the carina to the gastroesophageal junction, which reduced significantly the organ lumen (Fig. 4).

The patient currently receives budesonide $9 \mathrm{mg}$ PO OD and pantoprazole $40 \mathrm{mg} \mathrm{PO} \mathrm{OD}$, he is asymptomatic, with good response to treatment and has gained $20 \mathrm{~kg}$ since the last medical checkup (Fig. 6).

\section{Discussion}

The prevalence of EE has increased in the last few years, although it is unknown if this increment is due to a real increase of its incidence or its diagnosis. The classic clinical presentation of this pathology is dysphagia, regurgitation, heartburn, and food impaction $[9,10]$. EE is often confused with other pathologies with similar symptoms such as GERD or achalasia [7].

The diagnosis of EE is based on the symptoms, endoscopic findings, alterations in manometry tests and changes seen through image studies. However, the diagnosis is established when eosinophilic infiltration is greater than 15 eosinophils per HPF microscopic evaluation in biopsies of esophageal mucosa of patients who have previously received high doses of PPI and had no positive response [11].

It is important to emphasize that EE is not the only cause of eosinophilic infiltration; in fact, GERD is the most common cause of it. This is why, it is established that the finding of less than 5 eosinophils leads to the diagnosis of GERD, between 5 and 14 to a mixed condition and more than 15 to EE [10].

Zhao et al reported a case of a mural form of EE accompa-

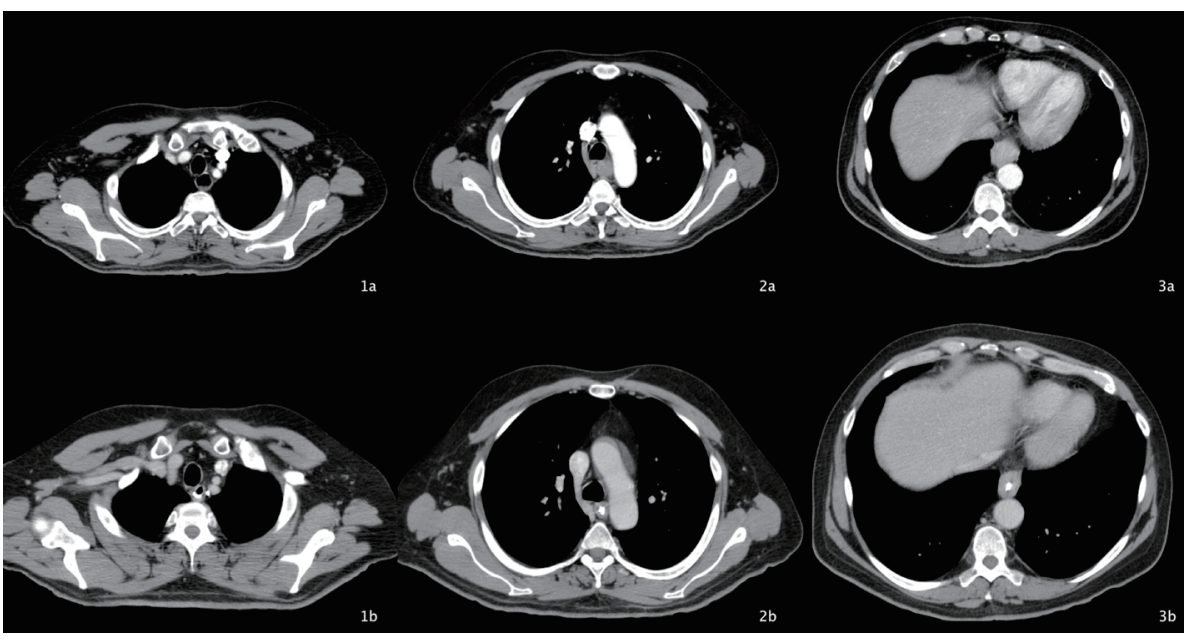

Figure 4. Double-contrasted thoracic CT scan during the hospitalization (a) and 4 months after symptoms remission (b). The images show the upper esophagus (1), mid esophagus (2) and lower esophagus (3). Note the diffuse thickening of the esophageal wall, which impairs the contrast passage through the mid and lower esophagus. 


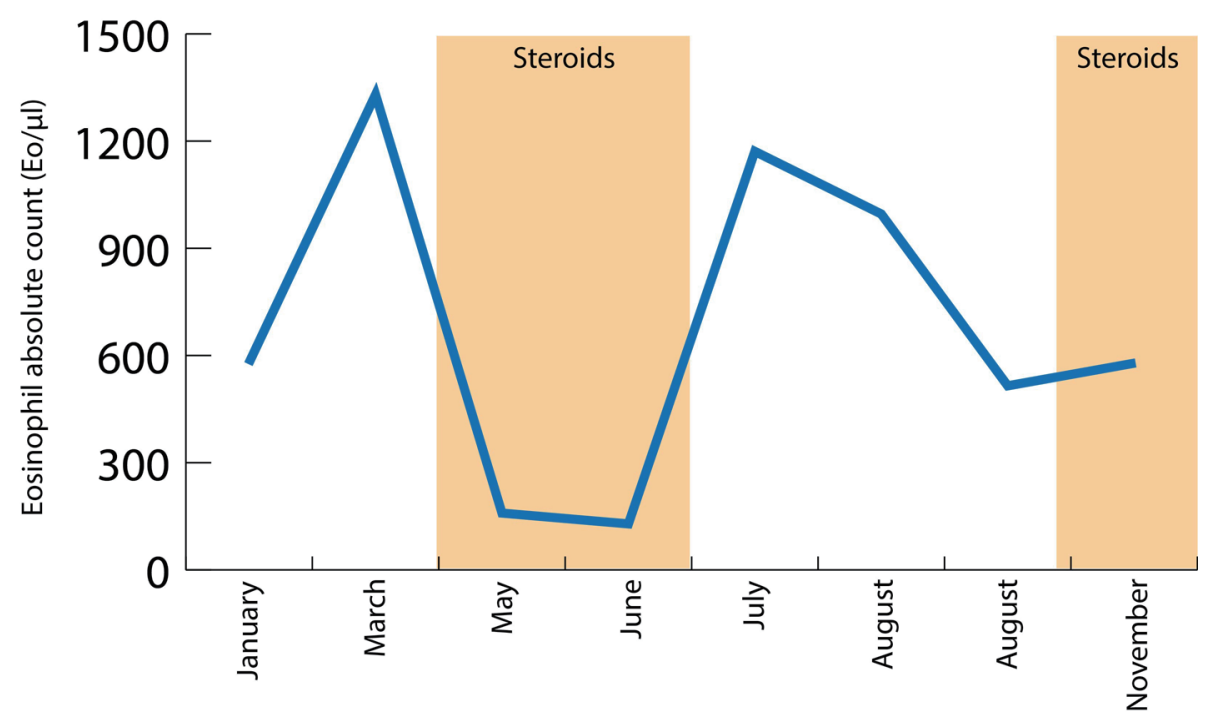

Figure 5. Eosinophil absolute count variation during patient's progression. Note that the threshold level for diagnosis of hypereosinophilic syndromes (> 1,500 eosinophils/ $\mu \mathrm{L})$ was never reached.

nied with squamous esophageal cancer. This patient presented dysphagia as the principal symptom. Endoscopic studies evidenced a tumor in the esophageal mucosa, which led to the resection of the esophagus. The biopsy of the resected tumor demonstrated that in addition to the cancer, eosinophilic infiltration in all the layers of the esophagus was also present, predominating in the muscular layer. Due to the histopathological changes, it could be determined that the eosinophilic infiltration preceded the neoplasia [10].

There are also other reported cases by Lucendo et al where patients, in addition to having the diagnostic criteria of EE, also presented motor impairments of the esophagus (66\%) and manometric changes such as secondary waves and abnormal peristalsis (one case), hypertension and incomplete lower esophageal sphincter relaxation (two cases) and incomplete upper esophageal sphincter relaxation (one case). In this study, it was presumed that these disorders could be associated to an eosinophilic infiltration of the muscular layer or the esophageal ganglion cells. Nevertheless, this could not be proven [11].

In our case, the patient presented dysphagia, regurgitation, heartburn and nausea. He also suffered an allergic cutaneous reaction 2 months before the onset of symptoms, but antigen sensitization tests and $\mathrm{IgE}$ concentration determination showed no abnormalities. The endoscopy showed changes in the esophageal mucosa suggestive of GERD and the esophageal manometry reported incomplete lower esophageal sphincter relaxation. However, the multiple biopsy samples obtained during the different endoscopies performed did not demonstrate an eosinophilic infiltration in the mucosa of the digestive tract. These findings led to treat the patient with high doses of PPI and to perform a Heller myotomy surgery, which were not effective.

After the Heller myotomy, fragments of the muscular layer were obtained. The histopathological analysis of these fragments revealed a severe eosinophilic infiltration of the muscular layer of the esophagus that suggested EE diagnosis, even though the mucosa did not have eosinophil infiltration.

Allergic conditions and involvement of other organs were ruled out. EAC was lower than 1,500 eosinophils/ $\mu \mathrm{L}$ in every $\mathrm{CBC}$ performed, thus excluding the presence of a hypereosinophilic syndrome (HES) [12]. The patient referred no symptoms suggestive of eosinophilic myositis or fasciitis (such as swelling and/or itching of the skin, or muscle weakness/pain); this is why no other studies were performed to rule out these pathologic entities.

The patient received the treatment established by the American Academy of Allergy Asthma and Immunology (AAAAI) [13], which consists on the administration of an intravenous steroid and then an oral one in order to manage the medical emergency and weight loss. Afterwards, the steroids were substituted by a low-dose non-intestinal-absorbable steroid (oral budesonide) as a maintenance treatment, although this change did not modify the patient's medical progress. The use of oral budesonide was effective.

Taking into account the latest advances made in the etiology and pathophysiology of EE, as well as the new variants that have been reported $[10,11]$, it is evident that there is a group of patients who probably have an eosinophilic disorder and do not meet the diagnostic criteria for any of those entities.

In this case, exclusive eosinophilic infiltration of the esophageal muscular layer, with evidence of esophageal dysfunction, was observed. The patient did not meet the diagnostic criteria for any of the differential diagnoses that were suggested (i.e. EE, GERD, HES, and GED), but the treatment with intravenous and oral steroids, as in EE, actually brought relief to the symptoms.

\section{Conflicts of Interests}

Authors have nothing to disclose. 


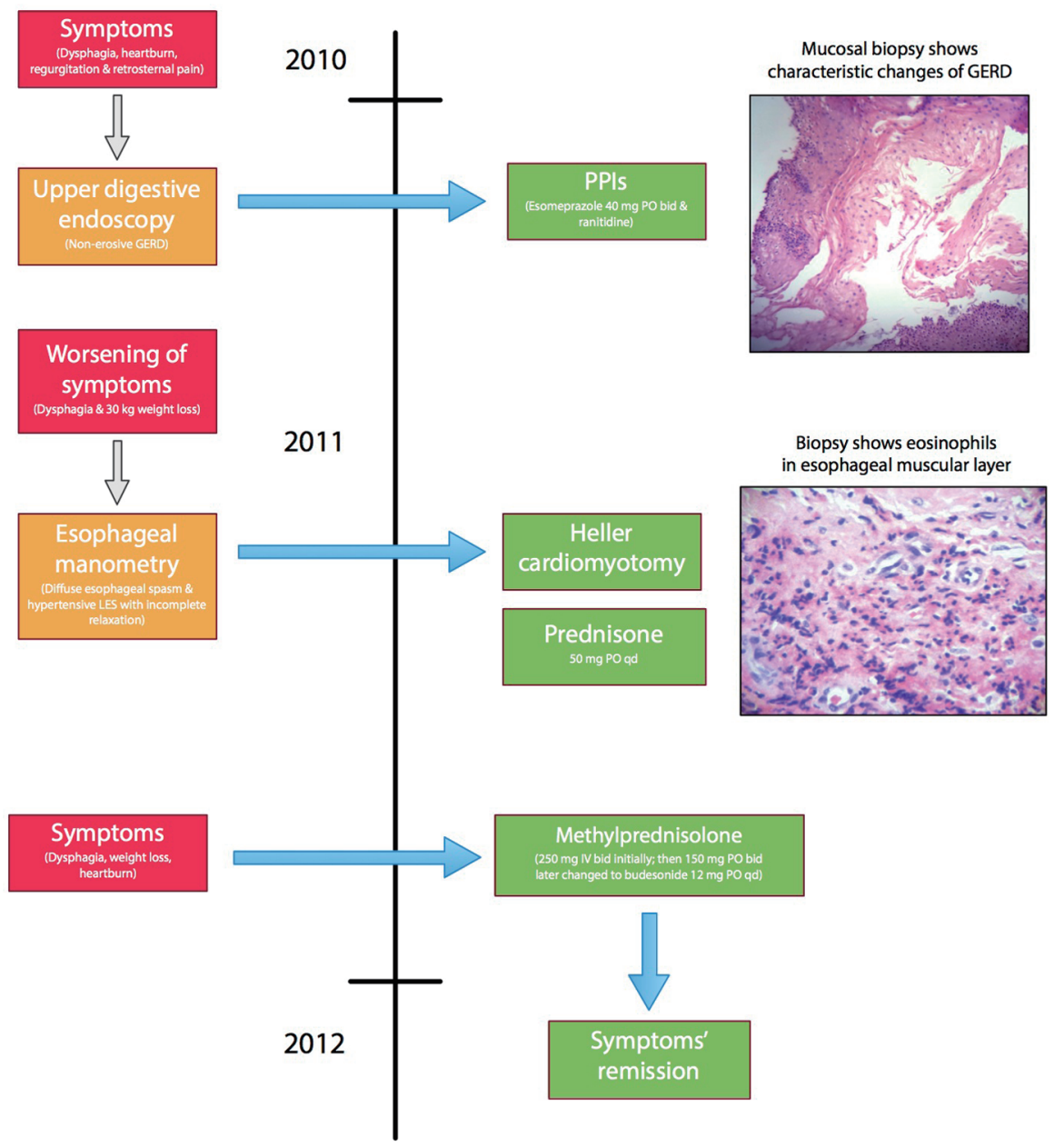

Figure 6. Case report timeline. Presented according to CARE guidelines.

\section{Author Contributions}

Jose Plaz provided the clinical and gastroenterologic studies' information of the patient as well as helped with the preparation of the manuscript. Renzo Di Natale provided the surgical data about the case report, as well as helped with the preparation and submission of the manuscript. Marisa Di Natale provided the histopathological information of the case report, as well as helped with the preparation of the manuscript. Andres Gonzalez provided the laboratory and other serologic tests information of the case report as well as helped with the preparation of the manuscript. Raquel Lander provided the CT and esophagogram's information of the case report as well as helped with the preparation of the manuscript.

\section{Abbreviations}

EE: eosinophilic esophagitis; GERD: gastroesophageal reflux disease; GED: gastrointestinal eosinophilic disorder; HES: hypereosinophilic syndrome; HPF: high-power field; PPI: proton pump inhibitor; CT: computed tomography; EAC: eosinophil absolute count; IgE: immunoglobulin E; AAAAI: American Academy of Allergy Asthma and Immunology

\section{References}

1. Attwood SE, Smyrk TC, Demeester TR, Jones JB. Esophageal eosinophilia with dysphagia. A distinct clinicopathologic syndrome. Dig Dis Sci. 1993;38(1):109-116.

2. Noel RJ, Putnam PE, Rothenberg ME. Eosinophilic esophagitis. N Engl J Med. 2004;351(9):940-941.

3. Cherian S, Smith NM, Forbes DA. Rapidly increasing prevalence of eosinophilic oesophagitis in Western Australia. Arch Dis Child. 2006;91(12):1000-1004.

4. Sampson HA. Food allergy: immunology of the GI mucosa towards classification and understanding of GI hypersensitivities. Pediatr Allergy Immunol. 2001;12(Suppl 14):7-9.

5. Blanchard C, Wang N, Stringer KF, Mishra A, Fulkerson PC, Abonia JP, Jameson SC, et al. Eotaxin-3 and a uniquely conserved gene-expression profile in eosino- 
philic esophagitis. J Clin Invest. 2006;116(2):536-547.

6. Nonesvki IT, Downs-Kelly E, Falk GW. Eosinophilic esophagitis: An increasingly recognized cause of dyphagia, food impaction and refractory heartburn. Clev Clin J Med. 2008;75:623-633.

7. Arora AS, Yamazaki K. Eosinophilic esophagitis: asthma of the esophagus? Clin Gastroenterol Hepatol. 2004;2(7):523-530.

8. DeVault KR, Castell DO. Updated guidelines for the diagnosis and treatment of gastroesophageal reflux disease. Am J Gastroenterol. 2005;100(1):190-200.

9. Genevay M, Rubbia-Brandt L, Rougemont AL. Do eosinophil numbers differentiate eosinophilic esophagitis from gastroesophageal reflux disease? Arch Pathol Lab Med. 2010;134(6):815-825.
10. Jingsheng Z, Yuncheng L, Yingye M, Hao L, Congyang L. The mural form of eosinophilic esophagitis is accompanied by superficial esophageal squamous cell carcinoma. Case Rep Pathol. 2012;2012:315428.

11. Lucendo AJ, Carrion G, Navarro M, et al. Esofagitis eosinofilica del adulto, causa emergente de disfagia. Presentacion de 9 casos. Rev Esp Enferm Dig. 2005;97(4):229239.

12. Roufosse FE, Goldman M, Cogan E. Hypereosinophilic syndromes. Orphanet J Rare Dis. 2007;2(37):1-12.

13. Liacouras CA, Furuta GT, Hirano I, Atkins D, Attwood SE, Bonis PA, Burks AW, et al. Eosinophilic esophagitis: updated consensus recommendations for children and adults. J Allergy Clin Immunol. 2011;128(1):3-20 e26; quiz 21-22. 